\title{
Numerical Treatment of Heat and Mass Transfer of MHD Flow of Carreau Fluid with Diffusion and Chemical Reaction through a Non Darcy Porous Medium
}

\author{
Mohamed Y. Abou-zeid* \\ Department of Mathematics, Faculty of Education, Ain Shams University, Heliopolis, Cairo, Egypt
}

\begin{abstract}
The numerical solutions of the system of non linear partial differential equations, which describe the unsteady flow of MHD non-Newtonian fluid with heat and mass transfer past a porous plate through a non-Darcy porous medium are obtained, taking into account the effects of viscous and ohmic dissipations and the chemical reaction. Finite difference method is used for solving the system of equations. The results of the velocity, temperature, concentration distributions, skin-friction, rate of heat and mass transfer are illustrated graphically for different values of physical parameters. Also, the stability condition is studied.
\end{abstract}

\section{1- INTRODUCTION}

Flow through porous media is very prevalent in nature, and therefore the study of flow through a porous medium has become of principle interest in many and engineering applications. Thermal and solutal transport by fluid flowing through a porous matrix is phenomenon of great interest from the theory and application point of view. Heat transfer in the case of homogenous fluid-saturated porous media has been studied with relation of different applications like dynamic of hot underground springs, terrestrial heat flow through aquifer, hot fluid and ignition front displacements in reservoir engineering, heat exchange between soil and atmosphere and heat exchanges with fluidized beds. Mass transfer in isothermal condition has been studied with applications to problems of mixing of fresh and salt water in a quifers, spreading of solules in fluidized beds and crystal washers, salt leaching in soils, etc. [1].

There has been a renewed interest in Darcy and nonDarcy flow in a non-Newtonian fluid saturated porous medium. This continuing interest due to its applications which including heat transfer enhancement, packed bed, chemical reactors, building insulation, enhanced oil recovery, food technology and filtration processes [2].

Darcy's law was the first model of transport processes in porous media. It's valid only for slow flows through porous media of relatively low permeability. The Darcy's law breaks down for flows at high velocity, in which inertia effects are no longer negligible. Forchheimer [3] proposed adding a velocity square term to the Darcy term that's to include the inertia effects in the momentum equation. For flow through a highly porous medium, the boundary frictional effect needs to be considered so that Brinkman [4] suggested adding a macroscopic viscous term. Thus, The Brinkman-Forchheimer- extended Darcy model (The non-Darcy effects) is the most general model governing flow in porous media. The

*Address correspondence to this author at the Department of Mathematics, Faculty of Education, Ain Shams University, Heliopolis, Cairo, Egypt; E-mail: master_math2003@yahoo.com
non-Darcy effects initially studied by Vafai [5]. The numerical solutions of the problem of heat and mass transfer of the pulsatile flow of magnetohydrodynamic nonNewtonian fluid (biviscosity fluid) through a porous medium between two permeable parallel plates was obtained by Mokhtar et al. [6]. Nakayama [7] investigated The Newtonian and non-Newtonian Couette flows through inelastic fluid saturated porous media due to a moving plate boundary. The steady MHD flow of an incompressible electrically conducting viscoelastic fluid through a porous medium between two porous parallel plates under the influence of a transverse magnetic field with heat and mass transfer in porous media analyzed by Eldabe and Sallam [8].

In his paper Elshehawy et al. [9] investigated the problem of the effect of porous medium peristaltic motion of a generalized Newtonian fluid. The non-Darcy mixed convection of a non-Newtonian fluid from a vertical isothermal plate embedded in a homogeneous porous medium in the presence of surface injection or suction investigated by Ibrahim et al. [10]. The non-Darcy mixed convection from a horizontal surface in a porous medium saturated with a power law fluid studied by Kumari and Nath [11].

In the present work, finite difference method is used to solve the system of non linear partial differential equations, which are obtained from the unsteady flow of an electrically conducting non-Newtonian (Carreau) fluid past a porous plate through a non-Darcy porous medium with heat and mass transfer in the presence of viscous and Joulean dissipations and chemical reaction effects. The plate is oscillating in its own plane with superimposed injection or suction. Numerical solutions are obtained for the velocity, temperature and concentration distributions is illustrated graphically. The Stability condition of the numerical method is studied.

\section{2- BASIC EQUATIONS}

The basic equations of the MHD flow of non-Newtonian fluid through non-Darcy porous media with heat and mass transfer using Carreau model [9] are: 


\section{Continuity Equation}

$\nabla \cdot \underline{V}=0$

\section{Momentum Equation}

$\rho \frac{d \underline{V}}{d t}=\nabla \cdot \Gamma+\underline{J} \times \underline{B}-\frac{\mu}{K} \underline{V}-\frac{C_{E} \rho}{\sqrt{K_{p}}}|\underline{V}| \underline{V}$

\section{Maxwell's Equations}

$\nabla \cdot \underline{B}=0$

$\nabla \times \underline{B}=\mu_{m} \underline{J}$

$\nabla \times \underline{E}=-\frac{\partial \underline{B}}{\partial t}$

$\underline{J}=\sigma(\underline{E}+\underline{V} \times \underline{B})$

\section{Heat Equation}

$\rho C_{p} \frac{d T}{d t}=k_{c} \nabla^{2} T+\Gamma_{i j} \frac{\partial V_{i}}{\partial x_{j}}+\frac{1}{\sigma}(\underline{J} \cdot \underline{J})+\frac{\rho D_{m} K_{T}}{C_{s}} \nabla^{2} C$

(4)

\section{Concentration Equation}

$\frac{d C}{d t}=D_{m} \nabla^{2} C+\frac{D_{m} K_{T}}{T_{m}} \nabla^{2} T$

The stress $\Gamma$ is defined by

$\Gamma_{i j}=\eta_{0}\left[1+\frac{(n-1)}{2} \lambda^{2} \dot{\gamma}^{2}\right] \dot{\gamma}_{i j}$

where $\dot{\gamma}=\sqrt{\frac{1}{2} \sum_{i} \sum_{j} \dot{\gamma}_{i j} \dot{\gamma}_{i j}}=\sqrt{\frac{1}{2} \prod_{\tilde{a}}}$ and the material time differentiation

$\frac{d(.)}{d t}=\frac{\partial(.)}{\partial t}+(\underline{V} \cdot \nabla)($.

\section{3- PROBLEM FORMULATION}

The flow of a non-Newtonian fluid that follows Carreau model on porous plate through a non-Darcy porous medium is considered. Choose the $\mathrm{x}$-axis along the plate and the $\mathrm{y}$ axis perpendicular to it. A uniform magnetic field $\underline{B}=\left(0, B_{0}, 0\right)$ is imposed normal to the plate (see Fig. (1)) and the induced magnetic field is negligible compared with the imposed field so that the magnetic Reynolds number is small. The quantities $\mu_{\mathrm{m}}$ and $\sigma$ are all constants throughout the flow field. The electric field $\underline{E}$ is assumed to be zero. In view of these assumptions, Eqn. (3b) can be ignored retaining the equation $\nabla . \underline{\mathrm{J}}=0$. For $\mathrm{t}<0$ fluid is at rest and for $\mathrm{t}>0$ the plate is moving periodically. From continuity equation (1) the velocity field $\underline{V}=\left(u(y ; t), V_{0}, 0\right), V_{0}<0$ is the suction velocity and $\mathrm{V}_{0}>0$ is the blowing velocity.

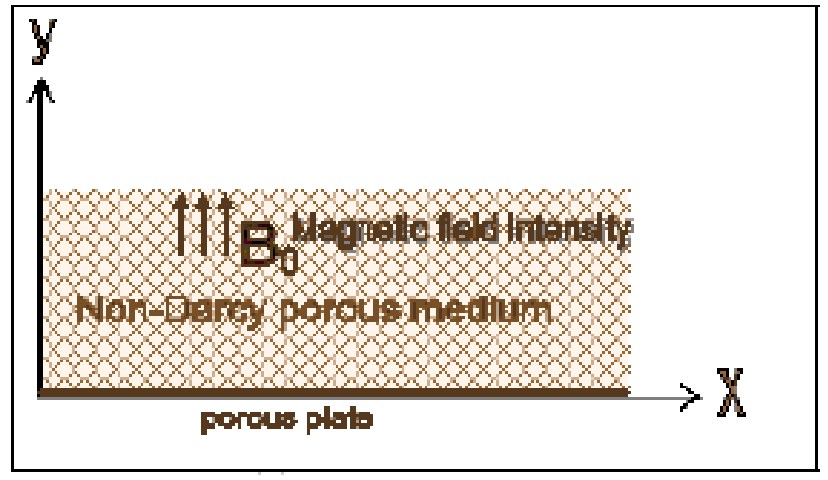

Fig. (1). Sketch of the problem.

The governing equations (1-6) can be simplified to

$$
\begin{aligned}
& \frac{\partial u}{\partial t}+V_{0} \frac{\partial u}{\partial y}=\frac{\eta_{0}}{\rho} \frac{\partial}{\partial y}\left[\left(1+\frac{n-1}{2} \lambda^{2}\left(\frac{\partial u}{\partial y}\right)^{2}\right) \frac{\partial u}{\partial y}\right] \\
& -\left(\frac{\sigma B_{0}^{2}}{\rho}+\frac{v}{K}\right) u-\frac{C_{E}}{\sqrt{K_{p}}} u^{2} .
\end{aligned}
$$

$\frac{\partial T}{\partial t}+V_{0} \frac{\partial T}{\partial y}=K \frac{\partial^{2} T}{\partial y^{2}}+$

$\frac{\eta_{0}}{\rho C_{p}}\left[\left(1+\frac{n-1}{2} \lambda^{2}\left(\frac{\partial u}{\partial y}\right)^{2}\right)\right]\left(\frac{\partial u}{\partial y}\right)^{2}+\frac{\sigma B_{0}^{2} u^{2}}{\rho C_{p}}$

$\frac{\partial C}{\partial t}+V_{0} \frac{\partial C}{\partial y}=D_{m} \frac{\partial^{2} C}{\partial y^{2}}-A\left(C-C_{\infty}\right)^{m}$

With initial and boundary conditions

at $t \leq 0: \quad u(y, t)=0, \quad T(y, t)=T_{\infty}, \quad C(y, t)=C_{\infty}$

$$
\text { at } t>0: \begin{aligned}
u(0, t) & =U_{0} e^{\left(\beta_{0}-I \omega\right) t}, T(0, t)=T_{\infty} \\
& +\left(T_{w}-T_{\infty}\right) e^{\left(\beta_{0}-I \omega\right) t} \\
C(0, t) & =C_{\infty}+\left(C_{w}-C_{\infty}\right) e^{\left(\beta_{0}-I \omega\right) t}
\end{aligned}
$$

$\lim _{y \rightarrow \infty} u(y, t)=0, \quad \lim _{y \rightarrow \infty} T(y, t)=T_{\infty}, \quad \lim _{y \rightarrow \infty} C(y, t)=C_{\infty}$

Introduce the dimensionless quantities:-

$$
\begin{aligned}
& \eta=\sqrt{\frac{\omega}{2 v}} y, \quad C_{0}=\frac{\beta_{0}}{\omega}, \quad \tau=\omega t, \quad d=\frac{V_{0}}{2 \sqrt{v \omega}} \\
& f=\frac{u}{U_{0}}, \quad \theta=\frac{T-T_{\infty}}{T_{w}-T_{\infty}}, \quad \psi=\frac{C-C_{\infty}}{C_{w}-C_{\infty}}
\end{aligned}
$$

using the dimensionless quantities (12) into the system of equations (7-11b) gives 


$$
\begin{aligned}
& \frac{\partial f}{\partial \tau}+\sqrt{2} d \frac{\partial f}{\partial \eta}=\hat{\alpha}\left[1+\frac{3(n-1)}{2} W^{2}\left(\frac{\partial f}{\partial \eta}\right)^{2}\right]\left(\frac{\partial^{2} f}{\partial \eta^{2}}\right) \\
& -\left(M+\frac{1}{D_{a}}\right) f-F_{s} f^{2}
\end{aligned}
$$

$\frac{\partial \theta}{\partial \tau}+\sqrt{2} d \frac{\partial \theta}{\partial \eta}=\frac{1}{P_{r}} \frac{\partial^{2} \theta}{\partial \eta^{2}}$

$+E_{c} \hat{\alpha}\left[1+\frac{3(n-1)}{2} W^{2}\left(\frac{\partial f}{\partial \eta}\right)^{2}\right]\left(\frac{\partial f}{\partial \eta}\right)^{2}+M E_{c} f^{2}$

$\frac{\partial \psi}{\partial \tau}+\sqrt{2} d \frac{\partial \psi}{\partial \eta}=\frac{1}{S_{c}} \frac{\partial^{2} \psi}{\partial \eta^{2}}-\delta \psi^{m}$

With initial and boundary conditions,

at $\quad \tau \leq 0: \quad f(\eta, \tau)=0, \quad \theta(\eta, t)=0, \quad \psi(\eta, \tau)=0$

at $\tau>0: f(0, \tau)=e^{\left(C_{0}-I\right) \tau}, \theta(0, \tau)=e^{\left(C_{0}-I\right) \tau}$,

$\psi(0, \tau)=e^{\left(C_{0}-I\right) \tau}$

$\lim _{\eta \rightarrow \infty} f(\eta, \tau)=0, \quad \lim _{\eta \rightarrow \infty} \theta(\eta, \tau)=0, \quad \lim _{\eta \rightarrow \infty} \psi(\eta, \tau)=0$

\section{4- METHOD OF SOLUTION}

We shall solve the system of non-linear partial differential equations numerically using the finite difference technique which is discussed by Soundalgekar [12] and so the system of equations (13-17b) yield

$$
\begin{aligned}
& \frac{f_{i}^{n+1}-f_{i}^{n}}{\Delta \tau}+\sqrt{2 d}\left(\frac{f_{i+1}^{n}-f_{i}^{n}}{\Delta \eta}\right)=\hat{\alpha}\left(\frac{f_{i+1}^{n}-2 f_{i}^{n}+f_{i-1}^{n}}{(\Delta \eta)^{2}}\right) \\
& \left(1+\frac{3(n-1)}{2} W^{2}\left(\frac{f_{i+1}^{n}-f_{i}^{n}}{\Delta \eta}\right)\right)-\left(M+\frac{1}{D_{a}}\right) f_{i}^{n}-F_{s}\left(f_{i}^{n}\right)^{2}
\end{aligned}
$$

$$
\begin{array}{r}
\frac{\theta_{i}^{n+1}-\theta_{i}^{n}}{\Delta \tau}+\sqrt{2 d}\left(\frac{\theta_{i+1}^{n}-\theta_{i}^{n}}{\Delta \eta}\right)= \\
P_{r}\left(\frac{\theta_{i+1}^{n}-2 \theta_{i}^{n}+\theta_{i-1}^{n}}{(\Delta \eta)^{2}}\right) \\
+M E_{c}\left(f_{i}^{n}\right)^{2}+E_{c} \hat{\alpha}\left(1+\frac{3(n-1)}{2} W^{2}\left(\frac{f_{i+1}^{n}-f_{i}^{n}}{\Delta \eta}\right)^{2}\right) \\
\left(\frac{f_{i+1}^{n}-f_{i}^{n}}{\Delta \eta}\right)^{2}
\end{array}
$$

$$
\begin{aligned}
& \frac{\psi_{i}^{n+1}-\psi_{i}^{n}}{\Delta \tau}+\sqrt{2 d}\left(\frac{\psi_{i+1}^{n}-\psi_{i}^{n}}{\Delta \eta}\right)=\frac{1}{S_{c}}\left(\frac{\psi_{i+1}^{n}-2 \psi_{i}^{n}+\psi_{i-1}^{n}}{(\Delta \eta)^{2}}\right) \\
& -\delta\left(\psi_{i}^{n}\right)^{m}
\end{aligned}
$$

With initial and boundary conditions,

$$
\begin{array}{ccc}
\text { at } \tau \leq 0: & f_{i}^{n}=0, & \theta_{i}^{n}=0, \quad \psi_{i}^{n}=0 \\
\text { at } \tau>0: & f_{i}^{n}=e^{\left.\left(C_{0}-I\right)(n \Delta \tau)\right)}, & \theta_{i}^{n}=e^{\left.\left(C_{0}-I\right)(n \Delta \tau)\right)}, \\
& \psi_{i}^{n}=e^{\left(C_{0}-I\right)(n(\Delta \tau))} & \text { at } \eta=0
\end{array}
$$

$f_{i}^{n} \rightarrow 0, \quad \theta_{i}^{n} \rightarrow 0, \quad \psi_{i}^{n} \rightarrow 0 \quad$ at $\eta \rightarrow \infty$

\section{1- Stability Condition of the Scheme}

The Von Neumann method is used to study the stability condition for the finite difference equations. Assume a Fourier component for $f_{i}^{n}, \theta_{i}^{n}$ and $\psi_{i}^{n}$ as,

$f_{i}^{n}=F(\tau) e^{I p(\Delta \eta) i}, \theta_{i}^{n}=\Theta(\tau) e^{I p(\Delta \eta) i}$ and $\psi_{i}^{n}=\Psi(\tau) e^{I p(\Delta \eta) i}$

where $\mathrm{p}$ is the wave number in $\mathrm{y}$-direction. Let $\varphi=\mathrm{p}(\Delta \eta)$ then,

$$
f_{i}^{n}=F(\tau) e^{I \varphi i}, \quad \theta_{i}^{n}=\Theta(\tau) e^{I \varphi i} \quad \text { and } \quad \psi_{i}^{n}=\Psi(\tau) e^{I \varphi i}
$$

Similarly,

$$
\begin{aligned}
f_{i \pm 1}^{n}=F(\tau) e^{I \phi(i \pm 1)}, \quad \theta_{1 \pm 1}^{n} & =\Theta(\tau) e^{I \phi(i \pm 1)} \quad \text { and } \\
\psi_{i \pm 1}^{n} & =\Psi(\tau) e^{I \phi(i \pm 1)}
\end{aligned}
$$

and

$$
f_{i}^{n+1}=F^{\prime}(\tau) e^{I \phi i}, \quad \grave{\mathrm{e}}_{i}^{n+1}=\grave{\mathrm{E}}^{\prime}(\tau) e^{I \phi i} \quad \text { and } \quad \varnothing_{i}^{n+1}=\emptyset^{\prime}(\tau) e^{I \phi i}
$$

Substituting equations (23-25) into equations (18-20) we get,

$$
\begin{array}{r}
\frac{F^{\prime}-F}{\Delta \tau}+\sqrt{2 d}\left(\frac{F\left(e^{I \phi}-1\right)}{\Delta \eta}\right)=\hat{\alpha}\left(1+\frac{3(n-1)}{2} W^{2}\left|\frac{f_{i+1}^{n}-f_{i}^{n}}{\Delta \eta}\right|^{2}\right), \\
\frac{F\left(e^{I \phi}-2+e^{-I \phi}\right)}{(\Delta \eta)^{2}}-\left(M+\frac{1}{D_{a}}\right) F-F_{s}\left|f_{i}^{n}\right| F
\end{array}
$$




$$
\begin{aligned}
& \frac{\Theta^{\prime}-\Theta}{\Delta \tau}+\sqrt{2 d}\left(\frac{\Theta\left(e^{I \phi}-1\right)}{\Delta \eta}\right)= \frac{1}{P_{r}}\left(\frac{\Theta\left(e^{I \phi}-2+e^{-I \phi}\right)}{(\Delta \eta)^{2}}\right) \\
&+M E_{c} F\left|f_{i}^{n}\right|+E_{c} \hat{\alpha}\left(1+\frac{3(n-1)}{2} W^{2}\left|\frac{f_{i+1}^{n}-f_{i}^{n}}{\Delta \eta}\right|\right) \\
& \\
& \mid \frac{f_{i+1}^{n}-f_{i}^{n}}{\Delta \eta}\left(\frac{F\left(e^{I \phi}-1\right)}{\Delta \eta}\right) \\
& \frac{\Psi^{\prime}-\Psi}{\Delta \tau}+\sqrt{2 d}\left(\frac{\Psi\left(e^{I \phi}-1\right)}{\Delta \eta}\right)= \frac{1}{S_{c}}\left(\frac{\Psi\left(e^{I \phi}-2+e^{-I \phi}\right)}{(\Delta \eta)^{2}}\right),(28) \\
&-\delta\left|\psi_{i}^{n}\right|^{m-1} \Psi
\end{aligned}
$$

The equations (26-28) can be written as,

$$
F^{\prime}=F\left[\begin{array}{c}
1-\sqrt{2 d}\left(\frac{\Delta \tau}{\Delta \eta}\right)\left(1-e^{I \phi}\right)+2 \hat{\alpha} \frac{(\Delta \tau)}{(\Delta \eta)^{2}}(\cos \phi-1) \\
\left(1+\frac{3(n-1)}{2} W^{2}\left|\frac{f_{i+1}^{n}-f_{i}^{n}}{\Delta \eta}\right|\right) \\
\left.-(\Delta \tau)\left(\mid M+\frac{1}{D_{a}}\right)+F_{s}\left|f_{i}^{n}\right|\right)
\end{array}\right],
$$

$$
\begin{aligned}
\Theta^{\prime}=F & {\left[\begin{array}{r}
E_{C} \hat{\alpha}\left(\frac{\Delta \tau}{\Delta \eta}\right)\left(e^{I \phi}-1\right)\left(1+\frac{3(n-1)}{2} W^{2}\left|\frac{f_{i+1}^{n}-f_{i}^{n}}{\Delta \eta}\right|^{2} \mid\right. \\
\left|\frac{f_{i+1}^{n}-f_{i}^{n} \mid}{\Delta \eta}\right|+(\Delta \tau) M E_{C}\left|f_{i}^{n}\right|
\end{array}\right], } \\
& +\Theta\left[1-\sqrt{2 d}\left(\frac{\Delta \tau}{\Delta \eta}\right)\left(1-e^{I \phi}\right)+\frac{2(\Delta \tau)}{P_{r}(\Delta \eta)^{2}}(\cos \phi-1)\right]
\end{aligned}
$$

$$
\Psi^{\prime}=\Psi\left[\begin{array}{c}
1-\sqrt{2 d}\left(\frac{\Delta \tau}{\Delta \eta}\right)\left(1-e^{I \phi}\right)+\frac{2(\Delta \tau)}{S_{C}(\Delta \eta)^{2}}(\cos \phi-1) \\
-\delta\left|\psi_{i}^{n}\right|^{m-1}
\end{array}\right]
$$

and equations (29-31),

$$
\begin{aligned}
& F^{\prime}=A_{1} F \\
& \Theta^{\prime}=A_{2} F+A_{3} \Theta \\
& \Psi^{\prime}=A_{4} \Psi
\end{aligned}
$$

where,

$$
\begin{aligned}
& A_{1}=\alpha_{1}+2 \alpha_{2} \alpha_{3}-\alpha_{4}, \\
& A_{2}=E c\left[\alpha_{2}\left(\frac{\Delta \tau}{\Delta \eta}\right)\left(e^{I \phi}-1\right)\left|\frac{\mathrm{f}_{\mathrm{i}+1}^{\mathrm{n}}-f_{i}^{n}}{\Delta \eta}\right|+(\Delta \tau) M\left|f_{i}^{n}\right|\right] \\
& A_{3}=\alpha_{1}+\frac{2}{P_{r}} \alpha_{3}, \\
& A_{4}=\alpha_{1}+\frac{2}{S c} \alpha_{3}-\delta|\Psi|^{m-1}, \\
& \alpha_{1}=1-\sqrt{2 d}\left(\frac{\Delta \tau}{\Delta \eta}\right)\left(1-e^{I \varphi}\right) \\
& \alpha_{2}=\hat{\alpha}\left(1+\frac{3(n-1)}{2} W^{2}\left|\frac{f_{i+1}^{n}-f_{i}^{n}}{\Delta \eta}\right|^{2}\right) \\
& \alpha_{3}=\frac{(\Delta \tau)}{(\Delta \eta)^{2}}(\cos \varphi-1) \\
& \alpha_{4}=(\Delta \tau)\left(\left(M+\frac{1}{D_{a}}\right)+F_{s}\left|f_{i}^{n}\right|\right)
\end{aligned}
$$

The equations (32-34) in matrix form can be expressed as follows,

$$
\left(\begin{array}{l}
F^{\prime} \\
\Theta^{\prime} \\
\Psi^{\prime}
\end{array}\right)=\left(\begin{array}{ccc}
A_{1} & 0 & 0 \\
A_{2} & A_{3} & 0 \\
0 & 0 & A_{4}
\end{array}\right)\left(\begin{array}{l}
F \\
\Theta \\
\Psi
\end{array}\right) ;
$$

the amplification factor is a matrix,

$$
\varepsilon=\left(\begin{array}{ccc}
A_{1} & 0 & 0 \\
A_{2} & A_{3} & 0 \\
0 & 0 & A_{4}
\end{array}\right)
$$

For stability, the modulus of each of the eigenvalue $\lambda_{m}$ of the amplification $\varepsilon$ must not exceed unity. The eigenvalues $\lambda$ are given by,

$$
\begin{aligned}
& \lambda_{1}=\alpha_{1}+2 \alpha_{2} \alpha_{3}-\alpha_{4} \\
& \lambda_{2}=\alpha_{1}+\frac{2}{P_{r}} \alpha_{3} \\
& \lambda_{3}=\alpha_{1}+\frac{2}{S c} \alpha_{3}-\delta|\Psi|^{m-1} .
\end{aligned}
$$

Hence the stability condition is:

$$
\left|\alpha_{1}+2 \alpha_{2} \alpha_{3}-\alpha_{4}\right| \leq 1
$$

The scheme is stable when inequality (35) is satisfied. The local truncation error by employing the procedure used in Smith [13] is $\mathrm{O}(\Delta \tau)+\mathrm{O}(\Delta \eta)$ and it tend to zero as $\Delta \tau \rightarrow 0$ and $\Delta \eta \rightarrow 0$. Hence, the scheme is compatible and then convergent, because compatibility and stability are necessary and sufficient conditions for convergence. 


\section{5- THE SKIN-FRICTION, HEAT AND MASS TRANSFER}

The skin-friction, heat and mass transfer in the non dimensional form can be defined as [2],

$\tau_{\omega}=\hat{\alpha}\left(\left(\frac{\partial^{2} f}{\partial \eta^{2}}\right)\left[1+\frac{3(n-1)}{2} W^{2}\left(\frac{\partial f}{\partial \eta}\right)^{2}\right]\right)_{\eta=0}$

$Q=-\left[\frac{\partial \theta}{\partial \eta}\right]_{\eta=0}$

$S_{t}=-\left[\frac{\partial \psi}{\partial \eta}\right]_{\eta=0}$

We can write Eqs. (36-38) by using finite difference method as follows:

$\tau_{\omega}=\hat{\alpha}\left(\frac{f_{2}^{n}-2 f_{1}^{n}+f_{0}^{n}}{(\Delta \eta)^{2}}\right)\left(1+\frac{3(n-1)}{2} W^{2}\left(\frac{f_{1}^{n}-f_{0}^{n}}{\Delta \eta}\right)^{2}\right)$
$Q=-\left(\frac{\theta_{1}^{n}-\theta_{0}^{n}}{\Delta \eta}\right)$

$S_{t}=-\left(\frac{\psi_{1}^{n}-\psi_{0}^{n}}{\Delta \eta}\right)$

\section{6- NUMERICAL RESULTS AND DISCUSSION}

In order to get an insight into the physical situation of the problem, we have computed numerical values of the velocity, temperature and concentration. They are obtained for various values of the parameters governing the problem. The numerical calculations were performed by using a mesh size $\Delta \eta=0.2$ and time step $\Delta \tau=\frac{\pi}{1000}$. Figs. (2-24) display the results for velocity, temperature and concentration profiles for different values of the parameters associated with the governing problem.

We observe that the velocity increases as Darcy number $\mathrm{D}_{\mathrm{a}}$, non dimensional parameter $\hat{\alpha}$ and parameter of influe-

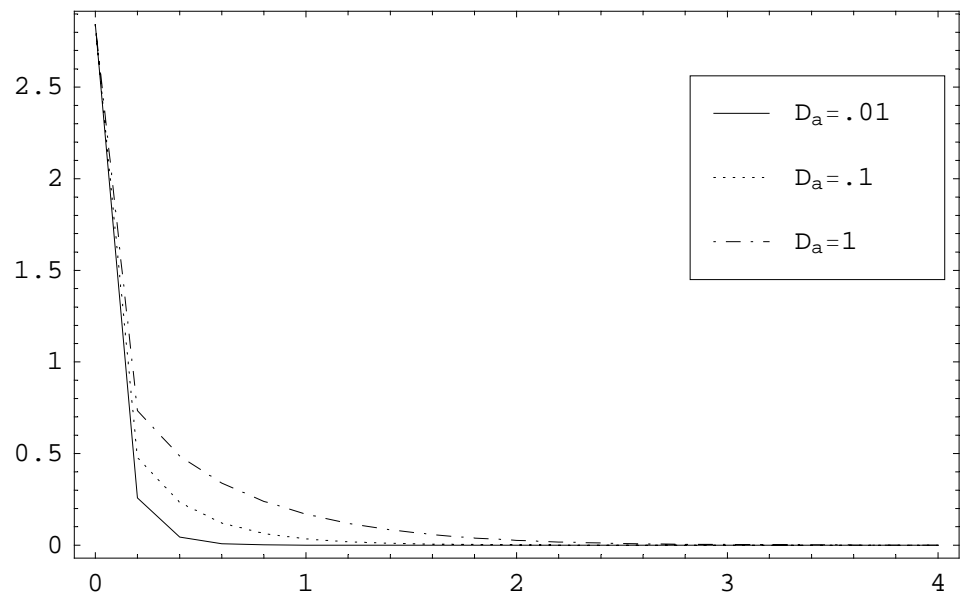

Fig. (2). The velocity distribution is plotted versus $y$, for $\mathrm{C}_{0}=2, \mathrm{~d}=0.15, \hat{\alpha}=1, \mathrm{n}=0.4, \mathrm{~W}=0.7, \mathrm{M}=1, \mathrm{Fs}=3, \mathrm{Pr}=3, \mathrm{Ec}=10, \mathrm{Sc}=5, \delta=1, \tau=\pi / 5$ and $\mathrm{m}=0.5$.

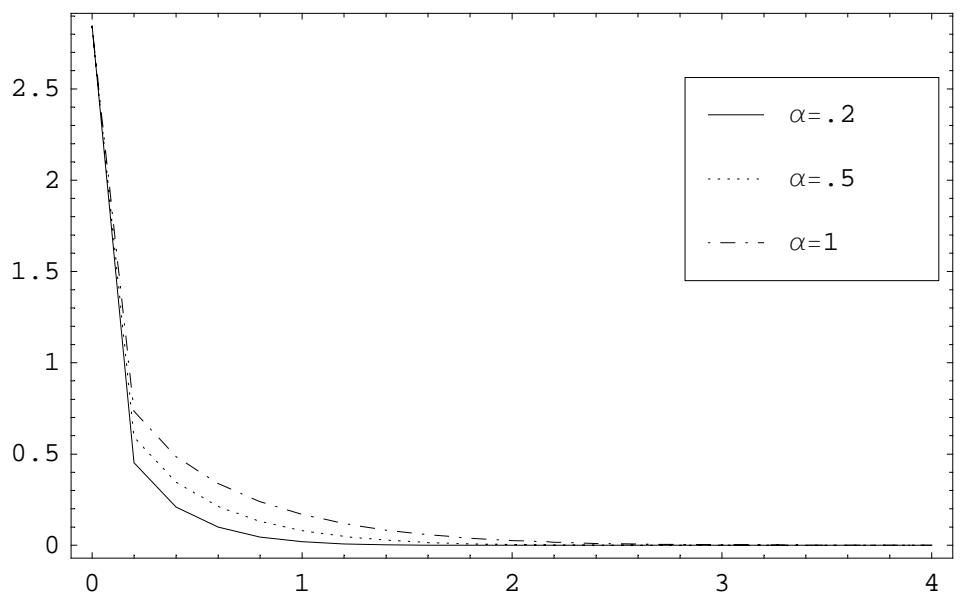

Fig. (3). The velocity distribution is plotted versus $y$, for $\mathrm{C}_{0}=2, \mathrm{~d}=0.15, \mathrm{n}=0.4, \mathrm{~W}=0.7, \mathrm{M}=1, \mathrm{Da}=0.01, \mathrm{Fs}=3, \mathrm{Pr}=3, \mathrm{Ec}=10, \mathrm{Sc}=5, \delta=1$, $\tau=\pi / 5$ and $\mathrm{m}=0.5$. 


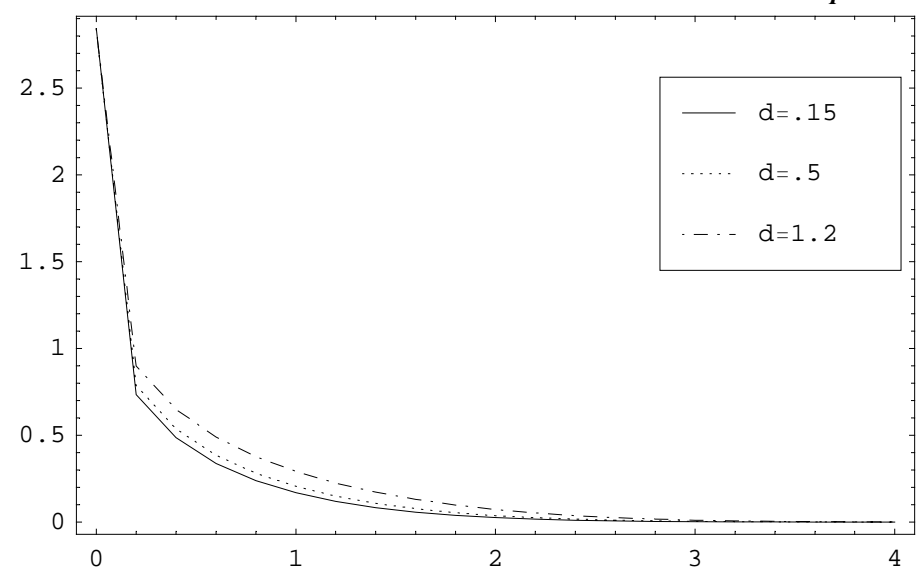

Fig. (4). The velocity distribution is plotted versus $y$, for $\mathrm{C}_{0}=2, \hat{\alpha}=1, \mathrm{n}=0.4, \mathrm{~W}=0.7, \mathrm{M}=1, \mathrm{Da}=0.01, \mathrm{Fs}=3, \mathrm{Pr}=3, \mathrm{Ec}=10, \mathrm{Sc}=5, \delta=1, \tau=\pi / 5$ and $\mathrm{m}=0.5$.

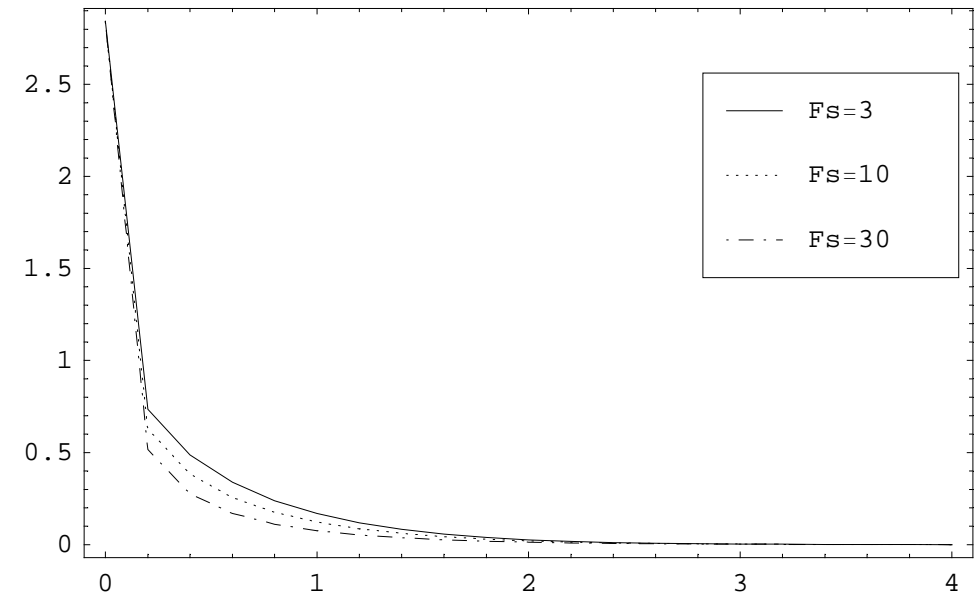

Fig. (5). The velocity distribution is plotted versus $y$, for $\mathrm{C}_{0}=2, \mathrm{~d}=0.15, \hat{\alpha}=1, \mathrm{n}=0.4, \mathrm{~W}=0.7, \mathrm{M}=1, \mathrm{Da}=0.01, \mathrm{Pr}=3, \mathrm{Ec}=10, \mathrm{Sc}=5, \delta=1$, $\tau=\pi / 5$ and $\mathrm{m}=0.5$.

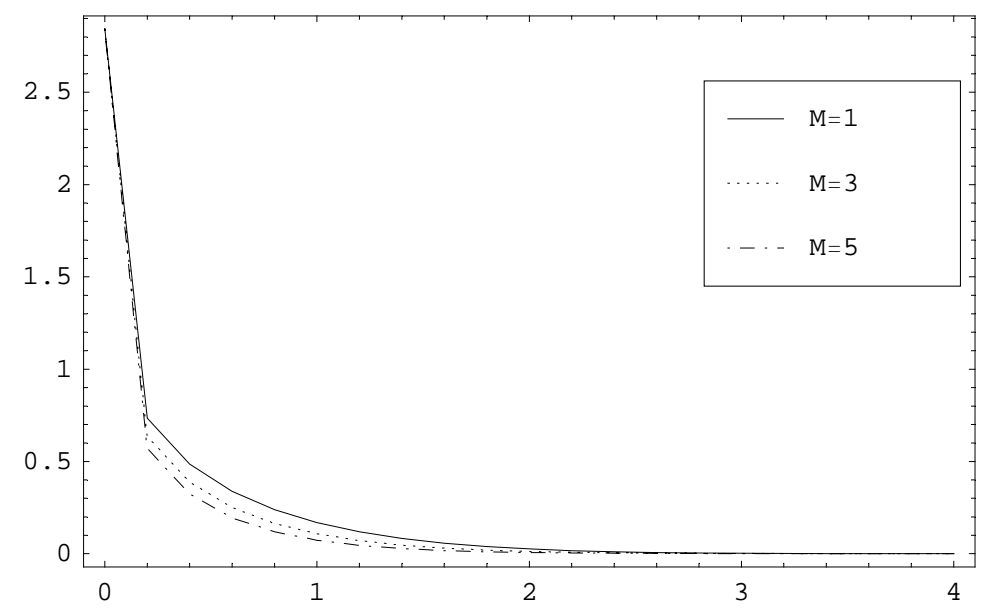

Fig. (6). The velocity distribution is plotted versus $y$, for $\mathrm{C}_{0}=2, \mathrm{~d}=0.15, \hat{\alpha}=1, \mathrm{n}=0.4, \mathrm{~W}=0.7, \mathrm{Da}=0.01, \mathrm{Fs}=3, \mathrm{Pr}=3, \mathrm{Ec}=10, \mathrm{Sc}=5, \delta=1$, $\tau=\pi / 5$ and $\mathrm{m}=0.5$.

nce of suction/blowing $d$ increase as shown in Figs. (2-4) but the velocity decreases as Forchheimer number $F_{s}$, Magnetic parameter $\mathrm{M}$ and Weissenberg number $\mathrm{W}$ increase as shown in Figs. (5-7). Figs. (8-9) show the effect of dimensionless power-law index $n$ and the time on velocity. It is observed that the velocity increases with increasing values of $n$ and $\tau$. Both of temperature and concentration increase as parameter of influence of suction/blowing $d$ and the time increase as shown in Figs. (10-13). The temperature decreases as
Forchheimer number Fs and Weissenberg number W increase as shown in Figs. (14-15). The temperature increases as Darcy number $\mathrm{D}_{\mathrm{a}}$, Eckert number Ec, dimensionless power-law index $n$, non dimensional parameter $\hat{\alpha}$ and Magnetic parameter $M$ increase as shown in Figs. (16-20). Also the temperature increases for $\eta<0.5$ but it decreases for $\eta>0.5$ as Prandtl number Pr increases as shown in Fig. (21). 


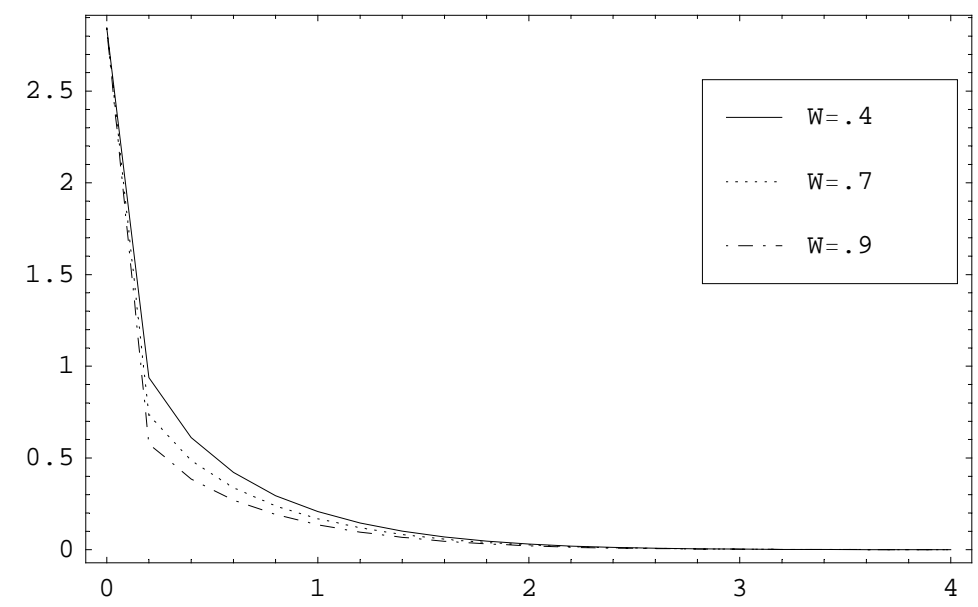

Fig. (7). The velocity distribution is plotted versus $y$, for $\mathrm{C}_{0}=2, \mathrm{~d}=0.15, \hat{\alpha}=1, \mathrm{n}=0.4, \mathrm{M}=1, \mathrm{Da}=0.01, \mathrm{Fs}=3, \mathrm{Pr}=3, \mathrm{Ec}=10, \mathrm{Sc}=5, \delta=1, \tau=\pi / 5$ and $\mathrm{m}=0.5$.

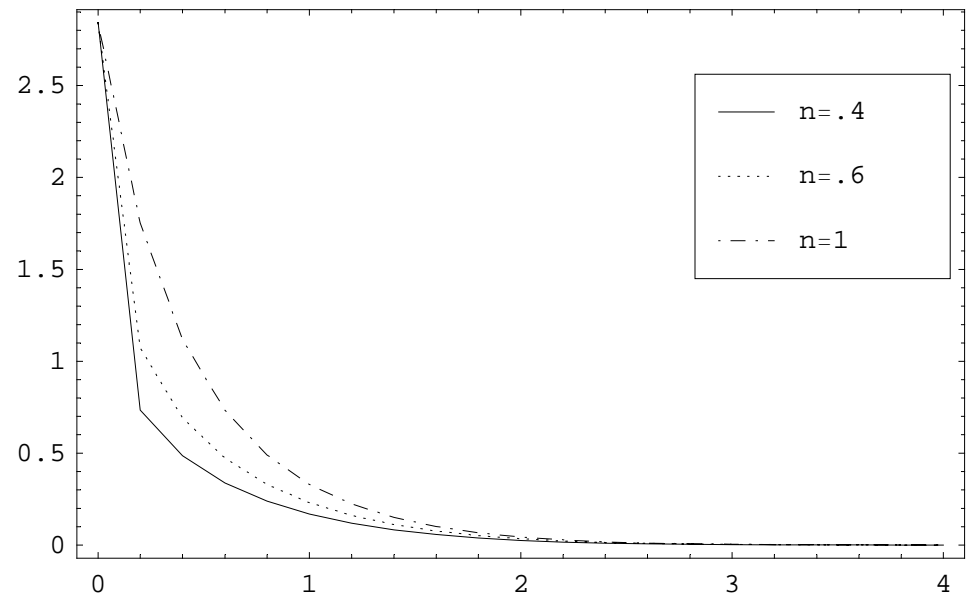

Fig. (8). The velocity distribution is plotted versus $y$, for $\mathrm{C}_{0}=2, \mathrm{~d}=0.15, \hat{\alpha}=1, \mathrm{~W}=0.7, \mathrm{M}=1, \mathrm{Da}=0.01, \mathrm{Fs}=3, \mathrm{Pr}=3, \mathrm{Ec}=10, \mathrm{Sc}=5, \delta=1, \tau=\pi / 5$ and $\mathrm{m}=0.5$.

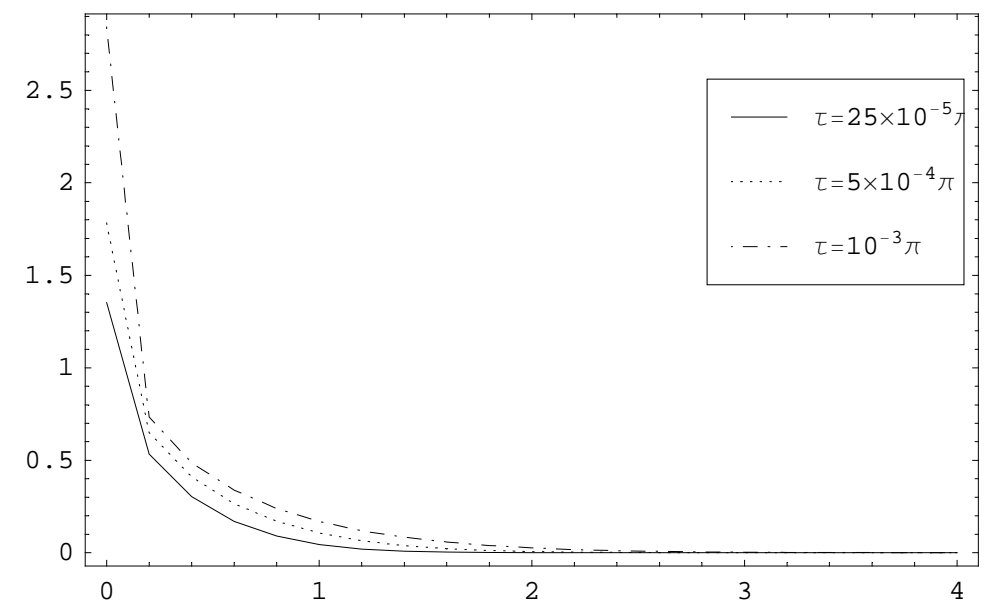

Fig. (9). The velocity distribution is plotted versus $y$, for $\mathrm{C}_{0}=2, \mathrm{~d}=0.15, \hat{\alpha}=1, \mathrm{n}=0.4, \mathrm{~W}=0.7, \mathrm{M}=1, \mathrm{Da}=0.01, \mathrm{Fs}=3, \mathrm{Pr}=3, \mathrm{Ec}=10, \mathrm{Sc}=5, \delta=1$ and $\mathrm{m}=0.5$. 


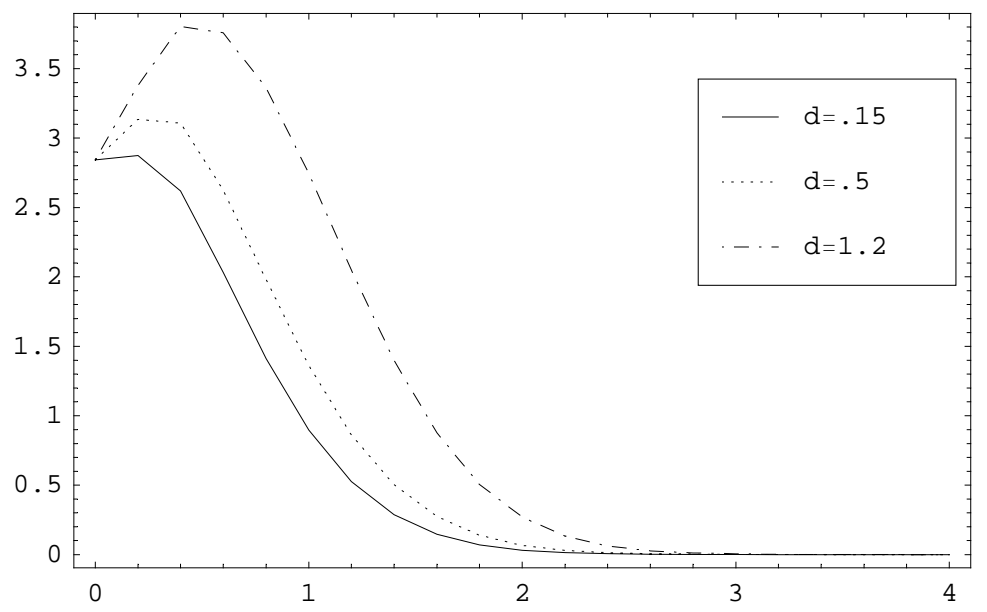

Fig. (10). The temperature distribution is plotted versus $y$, for $\mathrm{C}_{0}=2, \hat{\alpha}=1, \mathrm{n}=0.4, \mathrm{~W}=0.7, \mathrm{M}=1, \mathrm{Da}=0.01, \mathrm{Fs}=3, \mathrm{Pr}=3, \mathrm{Ec}=10, \mathrm{Sc}=5, \delta=1$, $\tau=\pi / 5$ and $\mathrm{m}=0.5$.

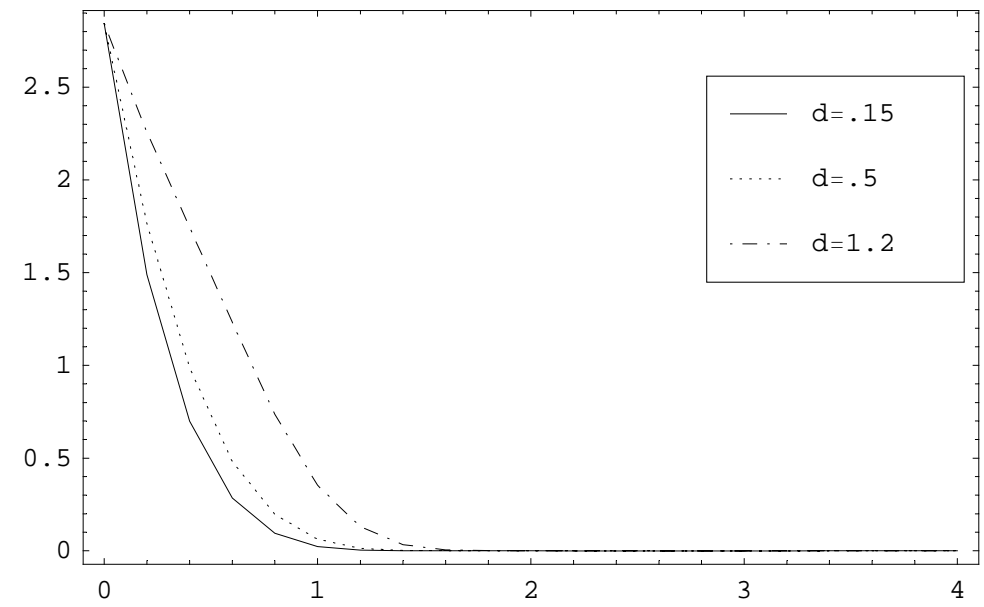

Fig. (11). The concentration distribution is plotted versus $y$, for $\mathrm{C}_{0}=2, \hat{\alpha}=1, \mathrm{n}=0.4, \mathrm{~W}=0.7, \mathrm{M}=1, \mathrm{Da}=0.01, \mathrm{Fs}=3, \mathrm{Pr}=3, \mathrm{Ec}=10, \mathrm{Sc}=5, \delta=1$, $\tau=\pi / 5$ and $m=0.5$.

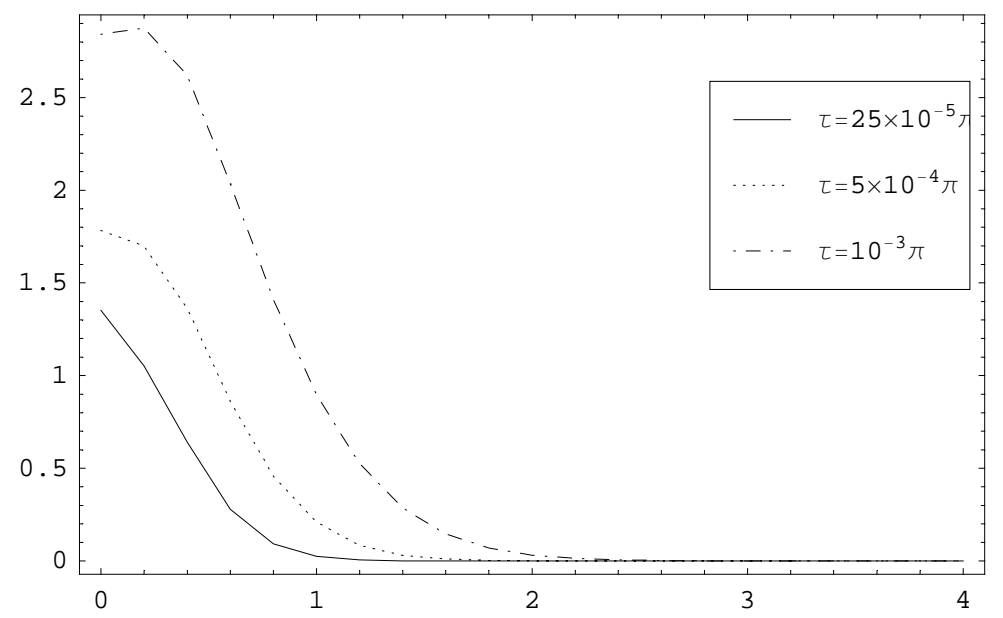

Fig. (12). The temperature distribution is plotted versus $y$, for $\mathrm{C}_{0}=2, \mathrm{~d}=0.15, \hat{\alpha}=1, \mathrm{n}=0.4, \mathrm{~W}=0.7, \mathrm{M}=1, \mathrm{Da}=0.01, \mathrm{Fs}=3, \mathrm{Pr}=3, \mathrm{Ec}=10, \mathrm{Sc}=5$, $\delta=1$, and $\mathrm{m}=0.5$. 


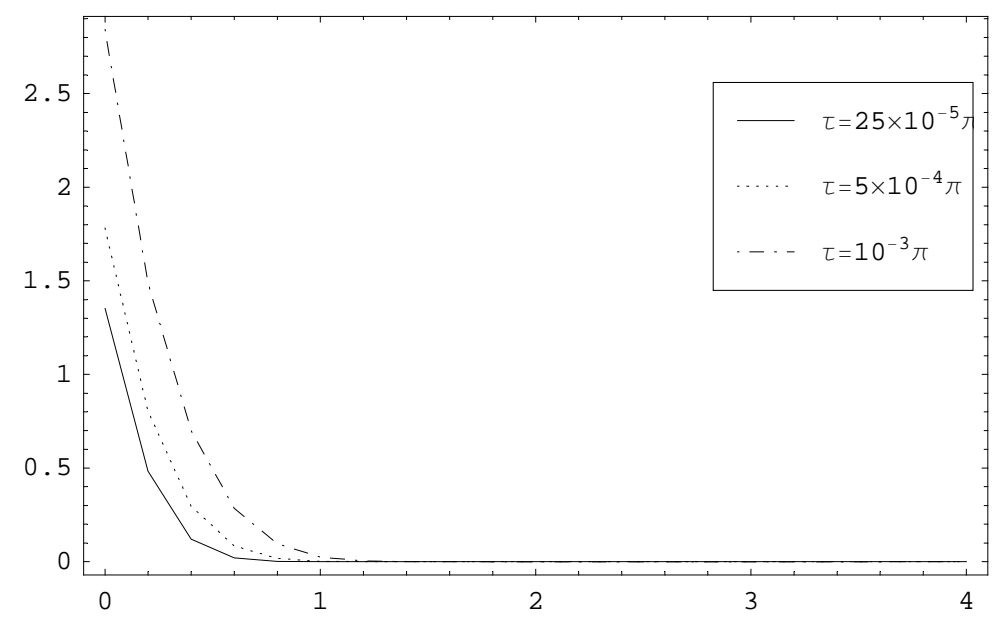

Fig. (13). The concentration distribution is plotted versus $y$, for $\mathrm{C}_{0}=2, \mathrm{~d}=0.15, \hat{\alpha}=1, \mathrm{n}=0.4, \mathrm{~W}=0.7, \mathrm{M}=1, \mathrm{Da}=0.01, \mathrm{Fs}=3, \mathrm{Pr}=3, \mathrm{Ec}=10$, $\mathrm{Sc}=5, \delta=1$ and $\mathrm{m}=0.5$.

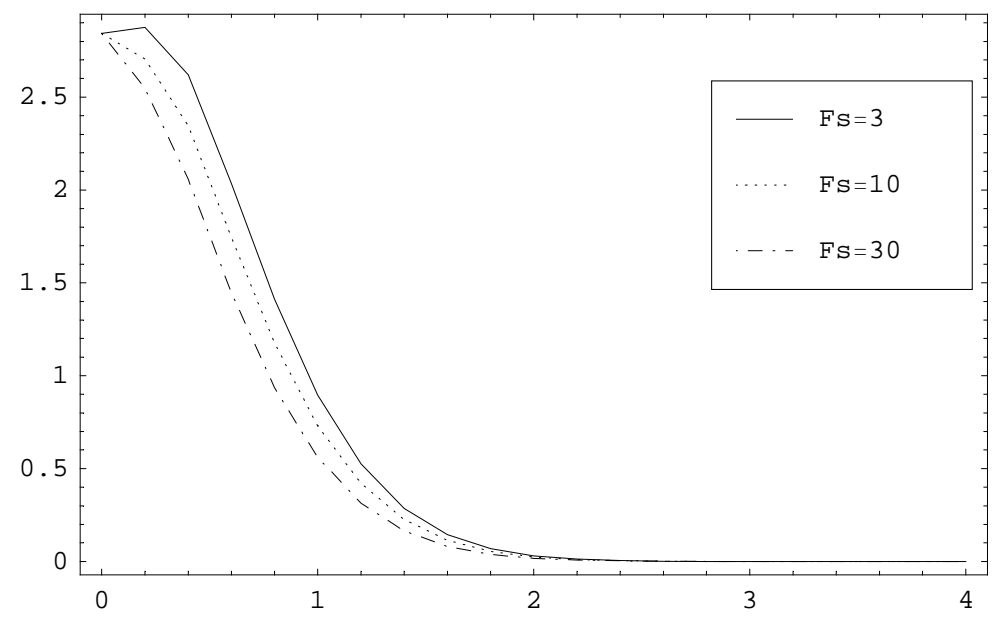

Fig. (14). The temperature distribution is plotted versus $y$, for $\mathrm{C}_{0}=2, \mathrm{~d}=0.15, \hat{\alpha}=1, \mathrm{n}=0.4, \mathrm{~W}=0.7, \mathrm{M}=1, \mathrm{Da}=0.01, \mathrm{Pr}=3, \mathrm{Ec}=10, \mathrm{Sc}=5, \delta=1$, $\tau=\pi / 5$ and $\mathrm{m}=0.5$.

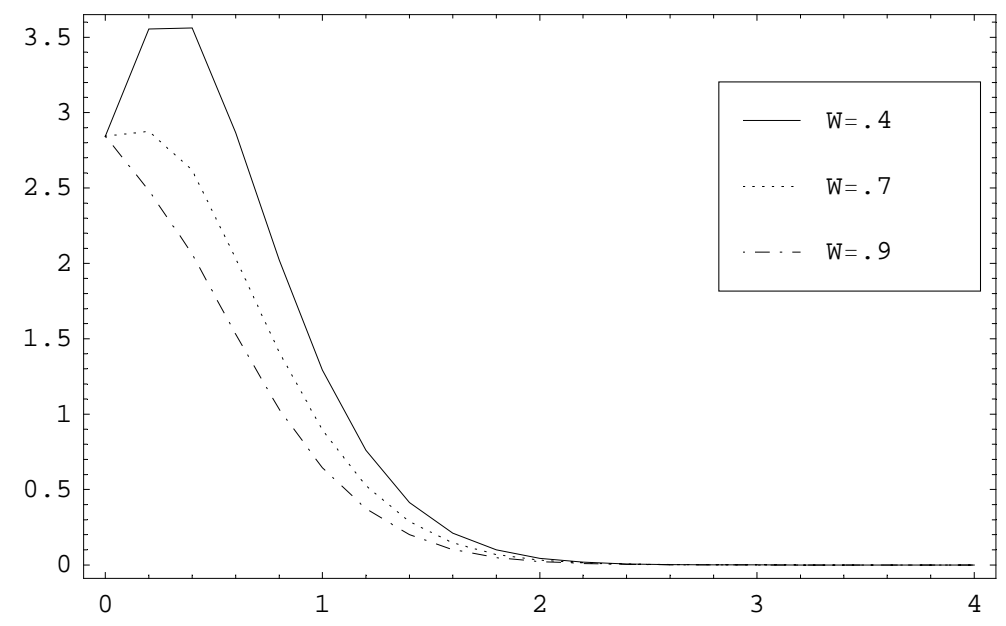

Fig. (15). The temperature distribution is plotted versus $y$, for $\mathrm{C}_{0}=2, \mathrm{~d}=0.15, \hat{\alpha}=1, \mathrm{n}=0.4, \mathrm{M}=1, \mathrm{Da}=0.01, \mathrm{Fs}=3, \mathrm{Pr}=3, \mathrm{Ec}=10, \mathrm{Sc}=5, \delta=1$, $\tau=\pi / 5$ and $\mathrm{m}=0.5$. 


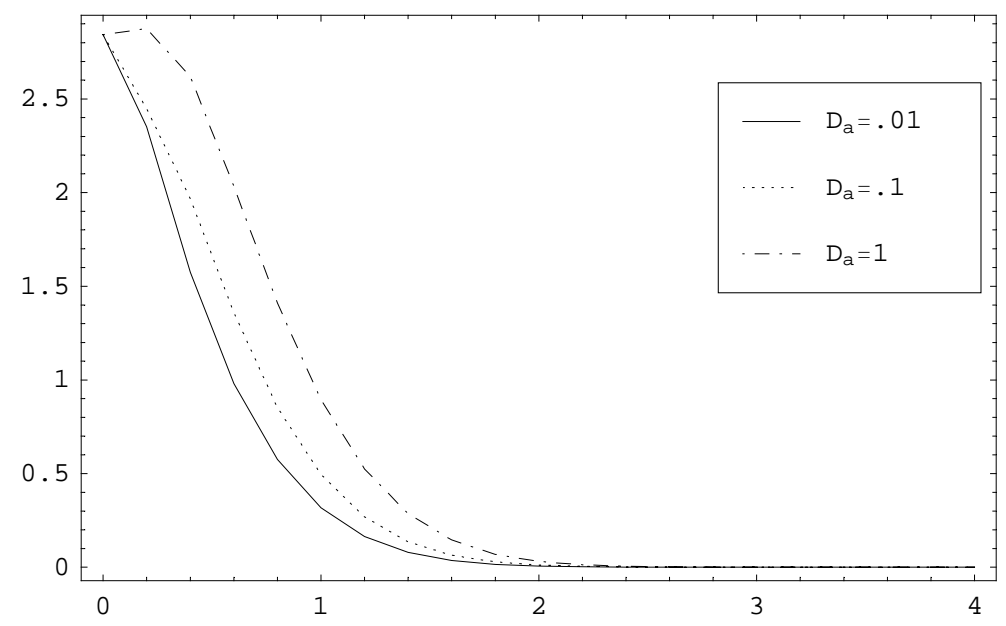

Fig. (16). The temperature distribution is plotted versus $y$, for $\mathrm{C}_{0}=2, \mathrm{~d}=0.15, \hat{\alpha}=1, \mathrm{n}=0.4, \mathrm{~W}=0.7, \mathrm{M}=1, \mathrm{Fs}=3, \mathrm{Pr}=3, \mathrm{Ec}=10, \mathrm{Sc}=5, \delta=1$, $\tau=\pi / 5$ and $\mathrm{m}=0.5$.

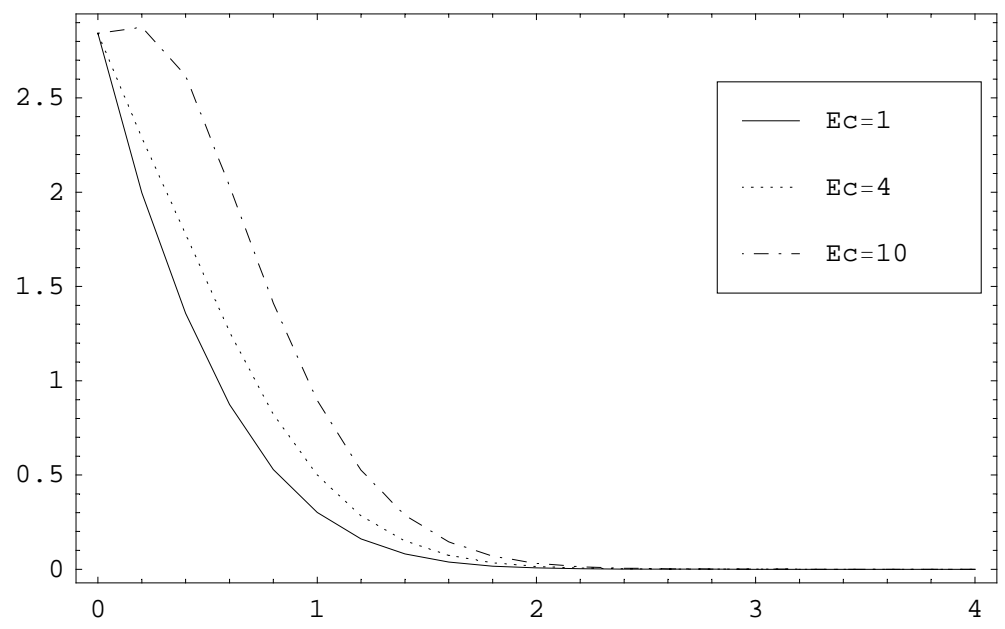

Fig. (17). The temperature distribution is plotted versus $y$, for $\mathrm{C}_{0}=2, \mathrm{~d}=0.15, \hat{\alpha}=1, \mathrm{n}=0.4, \mathrm{~W}=0.7, \mathrm{M}=1, \mathrm{Da}=0.01, \mathrm{Fs}=3, \mathrm{Pr}=3, \mathrm{Sc}=5, \delta=1$, $\tau=\pi / 5$ and $\mathrm{m}=0.5$.

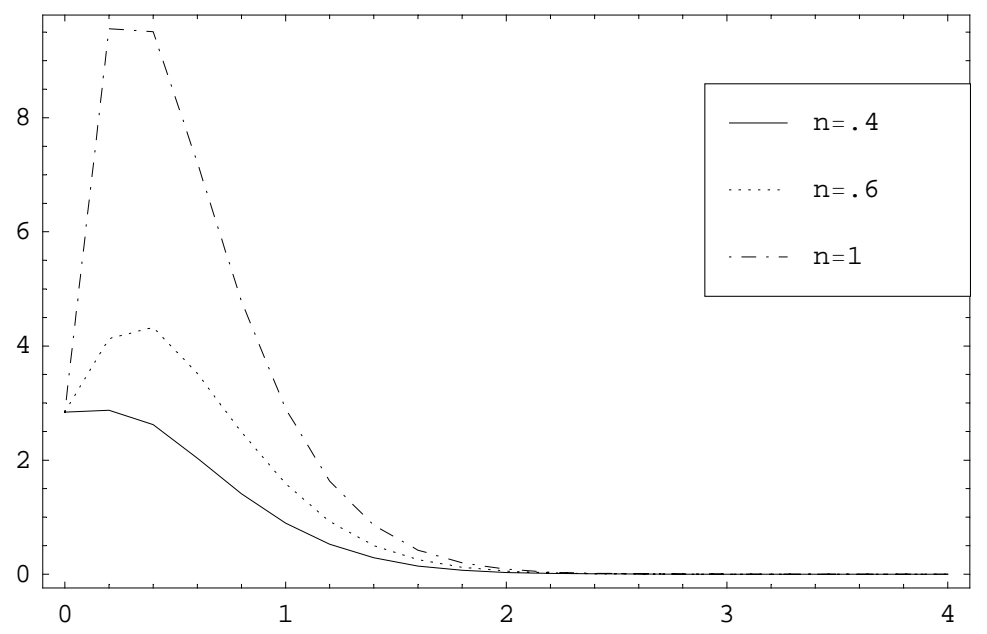

Fig. (18). The temperature distribution is plotted versus $y$, for $\mathrm{C}_{0}=2, \mathrm{~d}=0.15, \hat{\alpha}=1, \mathrm{~W}=0.7, \mathrm{M}=1, \mathrm{Da}=0.01, \mathrm{Fs}=3, \mathrm{Pr}=3, \mathrm{Ec}=10, \mathrm{Sc}=5, \delta=1$, $\tau=\pi / 5$ and $\mathrm{m}=0.5$. 


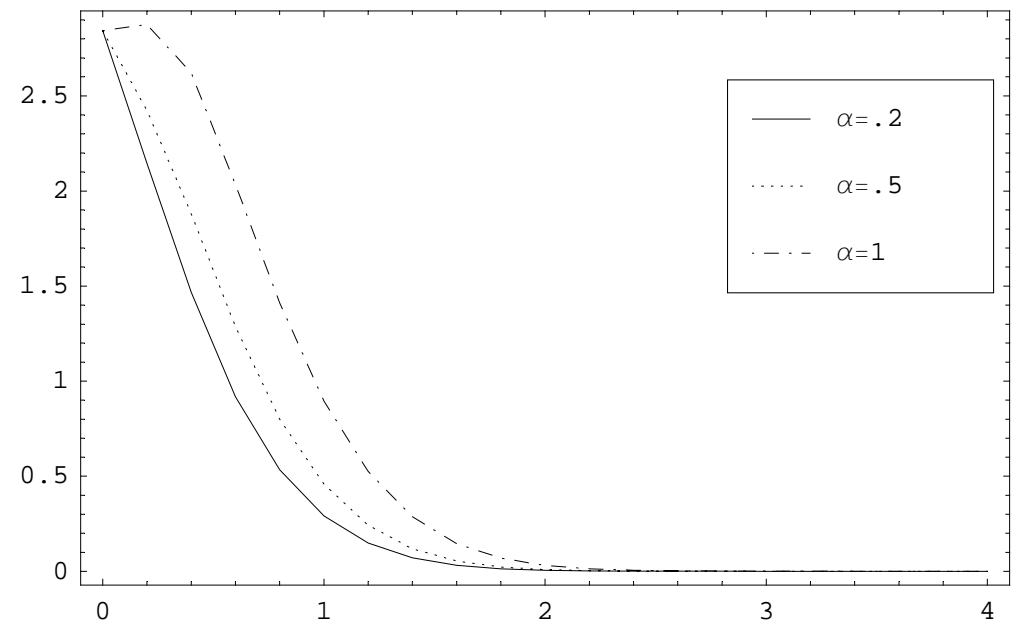

Fig. (19). The temperature distribution is plotted versus $y$, for $\mathrm{C}_{0}=2, \mathrm{~d}=0.15, \mathrm{n}=0.4, \mathrm{~W}=0.7, \mathrm{M}=1, \mathrm{Da}=0.01, \mathrm{Fs}=3, \mathrm{Pr}=3, \mathrm{Ec}=10, \mathrm{Sc}=5, \delta=1$ $\tau=\pi / 5$ and $\mathrm{m}=0.5$.

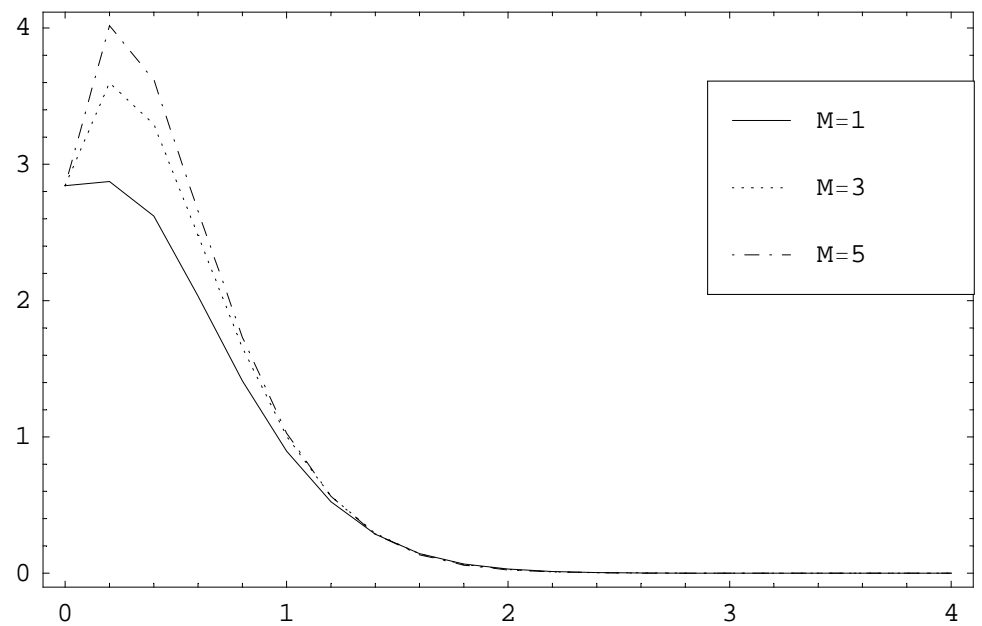

Fig. (20). The temperature distribution is plotted versus $y$, for $\mathrm{C}_{0}=2, \mathrm{~d}=0.15, \hat{\alpha}=1, \mathrm{n}=0.4, \mathrm{~W}=0.7, \mathrm{Da}=0.01, \mathrm{Fs}=3, \mathrm{Pr}=3, \mathrm{Ec}=10, \mathrm{Sc}=5, \delta=1$, $\tau=\pi / 5$ and $\mathrm{m}=0.5$.

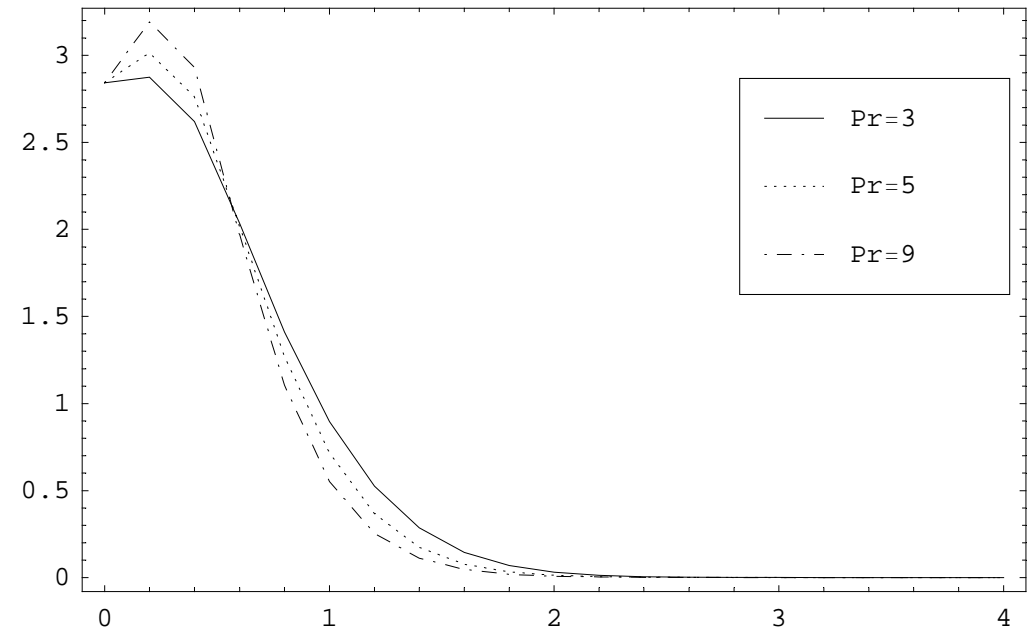

Fig. (21). The temperature distribution is plotted versus $y$, for $\mathrm{C}_{0}=2, \mathrm{~d}=0.15, \hat{\alpha}=1, \mathrm{n}=0.4, \mathrm{~W}=0.7, \mathrm{M}=1, \mathrm{Da}=0.01, \mathrm{Fs}=3, \mathrm{Ec}=10, \mathrm{Sc}=5, \delta=1$, $\tau=\pi / 5$ and $\mathrm{m}=0.5$.

Figs. (22-24) are graphed to illustrate the effects of the effects of chemical reaction $\delta$, Schmidt number Sc and reaction order $\mathrm{m}$ respectively. It is found that the concentration increases with increasing $\mathrm{m}$ but decreases as $\delta$ and Sc increase. 


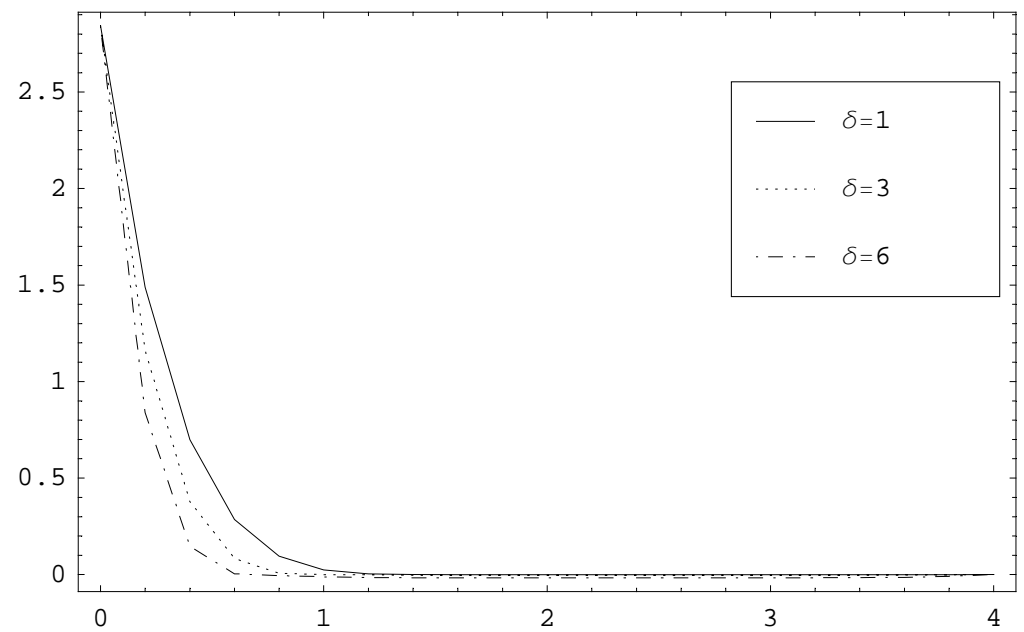

Fig. (22). The concentration distribution is plotted versus $y$, for $\mathrm{C}_{0}=2, \mathrm{~d}=0.15, \hat{\alpha}=1, \mathrm{n}=0.4, \mathrm{~W}=0.7, \mathrm{M}=1, \mathrm{Da}=0.01, \mathrm{Fs}=3, \mathrm{Pr}=3, \mathrm{Ec}=10$, $\mathrm{Sc}=5, \tau=\pi / 5$ and $\mathrm{m}=0.5$

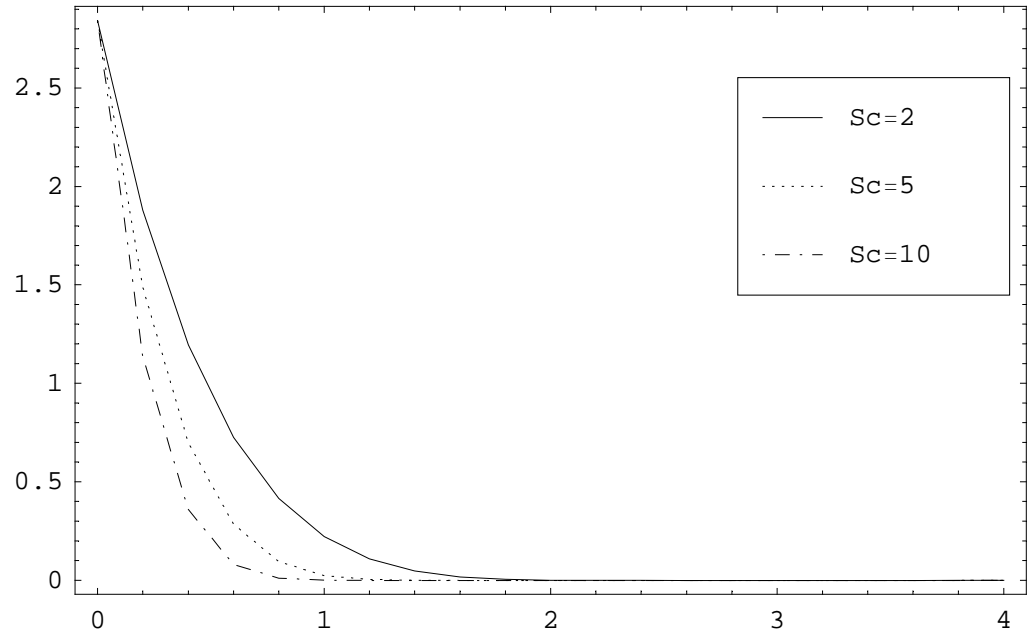

Fig. (23). The concentration distribution is plotted versus $y$, for $\mathrm{C}_{0}=2, \mathrm{~d}=0.15, \hat{\alpha}=1, \mathrm{n}=0.4, \mathrm{~W}=0.7, \mathrm{M}=1, \mathrm{Da}=0.01, \mathrm{Fs}=3, \mathrm{Pr}=3, \mathrm{Ec}=10, \delta=1$, $\tau=\pi / 5$ and $\mathrm{m}=0.5$.

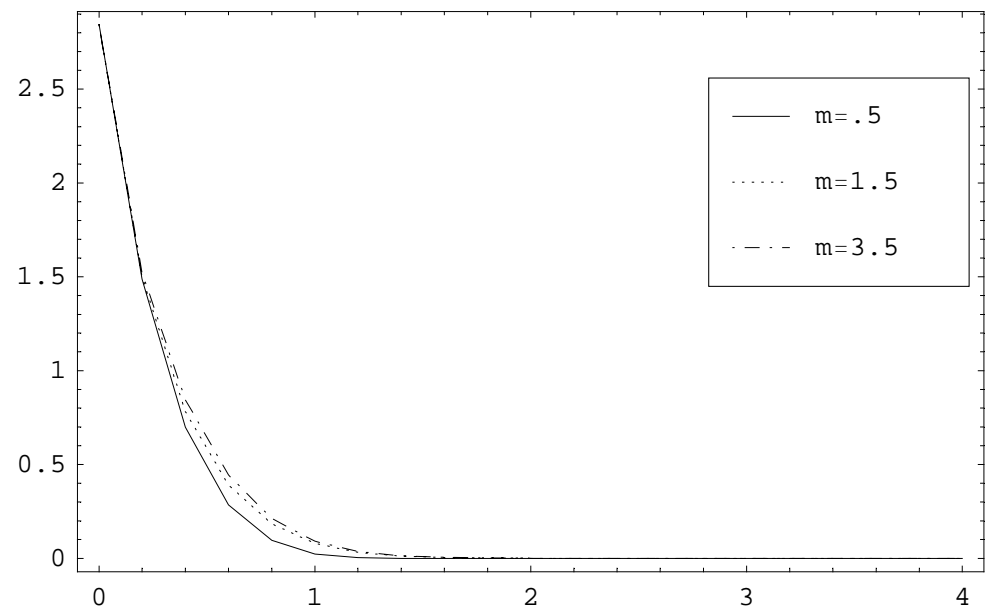

Fig. (24). The concentration distribution is plotted versus $y$, for $\mathrm{C}_{0}=2, \mathrm{~d}=0.15, \hat{\alpha}=1, \mathrm{n}=0.4, \mathrm{~W}=0.7, \mathrm{M}=1, \mathrm{Da}=0.01, \mathrm{Fs}=3, \mathrm{Pr}=3, \mathrm{Ec}=10$, $\mathrm{Sc}=5$ and $\delta=1$.

\section{CONCLUSION}

A numerical study using an explicit finite difference scheme is made to obtain the solution of the unsteady flow of MHD non-Newtonian fluid through a non-Darcy porous medium with heat and mass transfer. Also, we take into our consideration the effects of viscous and Ohmic dissipation 
and chemical reaction effects. In this study, a Carreau model is used as a non-Newtonian fluid and the DarcyForchheimer-Brinkman model is used to represent the fluid transport within the porous medium. The convergence of the scheme is studied using stability method. The effect of the problem's parameters such as non-Newtonian parameters $\hat{\alpha}$ and $\mathrm{W}$, magnetic parameter $\mathrm{M}$, Darcy number $\mathrm{D}_{\mathrm{a}}$, Forchheimer number $\mathrm{F}_{\mathrm{s}}$, Eckert number $\mathrm{E}_{\mathrm{c}}$, Schmidt number $\mathrm{S}_{\mathrm{c}}$, Prandtl number $\mathrm{P}_{\mathrm{r}}$, reaction order $\mathrm{m}$, chemical reaction $\delta$ and the parameter of influence of suction $\backslash$ blowing $d$ are discussed by a set of graphs. It is found that the velocity and temperature increase as Darcy number $\mathrm{D}_{\mathrm{a}}$ increases but they decrease as Forchheimer number $F_{s}$ increases. Also, it is found that the concentration decreases as Schmidt number Sc and chemical reaction $\delta$ increase.

For engineering purpose, the results of this problem are of great interest in petroleum applications as in reservoir of oil or gas flows, natural gas production and enhanced oil production. The flow of the Petroleum through the Porous ground represents an example of the motion of our fluid especially in the motion of the fluid in the earth's core. Also, there are many applications of this motion in many fields such Astrophysical, Plasma MHD, Metallurgical processes and Geophysical.

\section{NOMENCLATURE}

\begin{tabular}{|l|l|}
\hline A & reaction rate constant \\
\hline $\mathrm{B}$ & Total magnetic field \\
\hline $\mathrm{B}_{\mathrm{o} \backslash}$ & Constant \\
\hline $\mathrm{C}$ & Concentration of the species \\
\hline $\mathrm{C}_{\mathrm{E}}$ & Ergun constant \\
\hline $\mathrm{C}_{\mathrm{p}}$ & Specific heat at constant pressure \\
\hline $\mathrm{C}_{\mathrm{s}}$ & Concentration susceptibility \\
\hline $\mathrm{D}_{\mathrm{a}}$ & Darcy number \\
\hline $\mathrm{E}$ & Electric field \\
\hline $\mathrm{E}_{\mathrm{c}}$ & Eckert number $=\frac{U_{0}^{2}}{C_{p}\left(T_{w}-T_{\infty}\right)}$ \\
\hline $\mathrm{F}_{\mathrm{s}}$ & Forchheimer number $=\frac{C_{E} U_{0}}{\omega \sqrt{K_{p}}}$ \\
\hline $\mathrm{I}$ & \\
\hline $\mathrm{J}$ & $\sqrt{-1}$ \\
\hline $\mathrm{k}_{\mathrm{c}}$ & Current density \\
\hline $\mathrm{K}_{\mathrm{p}}$ & Thermal conductivity \\
\hline $\mathrm{K}_{\mathrm{T}}$ & Thermal diffusion ratio \\
\hline $\mathrm{m}$ & \\
\hline & \\
\hline & \\
\hline & \\
\hline & \\
\hline & \\
\hline & \\
\hline
\end{tabular}

\begin{tabular}{|c|c|}
\hline $\mathrm{n}$ & Dimensionless power-lawindex \\
\hline $\mathrm{P}_{\mathrm{r}}$ & Prandtl number $\frac{2 \nu}{K_{T}}$ \\
\hline $\mathrm{S}_{\mathrm{c}}$ & Schmidt number $=\frac{2 \nu}{D_{m}}$ \\
\hline $\mathrm{t}$ & Time \\
\hline $\mathrm{T}$ & Temperature of the fluid \\
\hline $\mathrm{T}_{\mathrm{m}}$ & The mean temperature \\
\hline $\mathrm{U}_{\mathrm{o}}$ & The reference velocity or free stream velocity \\
\hline$\underline{\mathrm{V}}$ & Velocity vector $=\left(\mathrm{u}(\mathrm{y}, \mathrm{t}), \mathrm{V}_{\mathrm{o}}, 0\right)$ \\
\hline W & Weissenberg number $=\lambda U_{0} \sqrt{\frac{\omega}{2 \nu}}$ \\
\hline Gree & lbols \\
\hline$\hat{\alpha}$ & Non dimensional parameter $=\frac{\eta_{0}}{2 \mu}$ \\
\hline$\beta_{\mathrm{o}}$ & The acceleration / deceleration parameter \\
\hline$\delta$ & Chemical reaction $=\mathrm{A} r_{e}\left(\mathrm{C}_{1}-\mathrm{C}_{2}\right)^{\mathrm{m}-1} / U_{0}$ \\
\hline$\eta_{0}$ & Zero-shear-rate viscosity \\
\hline$\dot{\gamma}_{i j}$ & Strain-rate tensor \\
\hline $\mathrm{K}$ & Thermal diffusivity $=\frac{k_{c}}{\rho C_{p}}$ \\
\hline$\lambda$ & Time constant \\
\hline$\mu$ & Viscosity \\
\hline$\mu_{\mathrm{m}}$ & Magnetic permeability \\
\hline$v$ & Kinematic viscosity $=\frac{\mu}{\rho}$ \\
\hline$\omega$ & Frequency of the oscillating plate \\
\hline$\prod_{\tilde{a}}$ & Second invariant of strain-rate tensor $\dot{\gamma}_{i j}$ \\
\hline$\rho$ & Density of the species \\
\hline$\sigma$ & Electrical conductivity of the fluid \\
\hline$\Gamma_{\mathrm{ij}}$ & Stress tensor in the Carreau model. \\
\hline \multicolumn{2}{|c|}{ Superscripts and subscripts } \\
\hline$\infty$ & Free stream condition \\
\hline $\mathrm{W}$ & Wall or plate condition. \\
\hline
\end{tabular}

\section{REFERENCES}

[1] Murthy SN. Effect of double dispersion on mixed convection heat and mass transfer in a non-Darcy porous medium. ASME J Heat Transfer 2000; 122: 476 . 
[2] Amany SA. Numerical treatment for the solutions of problems of non- Newtonian fluids flow through different geometric surfaces. M. Sc. Thesis. Faculty of Education. Roxy, Egypt: Ain Shams University 2007.

[3] Forchheimer P. Wasserbewegung Durch Boden. Zeit ver Deut Ing 1901; 45: 1782 .

[4] Birnkman HC. A calculation of the viscous force exerted by a flowing fluid on a Dense swarm of particles. Appl Sci Res 1974; AI: 27.

[5] Vafai K. Boundary and Inertia effects on flow and heat transfer in porous media. Int J Heat Mass Transfer 1981; 24: 195.

[6] Mokhtar AA, Eldabe NT, and Abou-zeid MY. Numerical study of pulsatile MHD non-Newtonian fluid flow with heat and mass transfer through a porous medium between two permeable parallel plates. Ind J Mech Cont Math Sci 2006; 1(1): 1.

[7] Nakayama A. Non-Darcy Couette flow in a porous medium filled with an Inelastic non-Newtonian fluid. Trans ASME 1992; 114: 642 .
[8]

Eldabe NT, Sallam SN. Non-Darcy Couette flow through a porous medium of magnetohydrodynamic visco-elastic fluid with heat and mass transfer. Can J Phys 2005; 83: 1243.

[9] El-shehawy EF, Ayman MF, Elsayed ME. Peristaltic motion of a generalized Newtonian fluid though a porous medium. J Phys Soc Jpn 2000; 69(2): 401.

[10] Ibrahim FS, Abdel Gaid SM, Subba R, Gorla R. Non-Darcy mixed convection flow along a vertical plate embedded in a nonNewtonian fluid saturated porous medium with surface mass transfer. Int J Num Method Heat Fluid Flow 2000; 10: 397.

[11] Kumari M, Nath G. Non-Darcy mixed convection in power-law fluids along a non-isothermal horizontal surface in a porous medium. Int J Eng Sci 2004; 42: 353.

[12] Soundalgekar VM. Finite difference analysis of transient free convection with mass transfer on an isothermal vertical flat plate. Int J Eng Sci 1981; 19: 757.

[13] Smith GD. Numerical solution of partial differential equations. New York: Oxford University press 1985.

Received: November 10, 2008

Revised: March 25, 2009

Accepted: June 01, 2009

(c) Mohamed Y. Abou-zeid; Licensee Bentham Open.

This is an open access article licensed under the terms of the Creative Commons Attribution Non-Commercial License (http://creativecommons.org/licenses/by$\mathrm{nc} / 3.0 /$ ), which permits unrestricted, non-commercial use, distribution and reproduction in any medium, provided the work is properly cited. 